\title{
Picosecond laser machining in the bulk of transparent dielectrics: critical comparison with fs-laser direct writing Costantino Corbari ${ }^{1, *}$, Audrey Champion ${ }^{2}$, Mindaugas Gecevičius ${ }^{1}$, Martynas Beresna ${ }^{1}$, Matthieu Lancry ${ }^{3}$, Bertrand Poumellec ${ }^{3}$, Yves Bellouard ${ }^{2}$, Peter G. Kazansky ${ }^{1}$ \\ ${ }^{1}$ Optoelectronics Research Centre, University of Southampton, SO17 1BJ, United Kingdom \\ ${ }^{2}$ Mechanical Engineering Dept. Eindhoven University of Technology, Eindhoven, The Netherlands \\ ${ }^{3}$ Institut de Chimie Moléculaire et des Matériaux d'Orsay, UMR 8182, Université de Paris Sud 11, Bâtiment 410, 91405 Orsay, France coc@orc.soton.ac.uk
}

\begin{abstract}
Picosecond lasers for bulk machining of transparent dielectrics are assessed as an alternative to fs-lasers. Nanogratings and micro-channels by selective etching are demonstrated. Scattering and inhomogeneous etching are challenges yet to be solved.

OCIS codes: (140.3390) Laser materials processing; (140.7090) Ultrafast lasers; (160.6030) Silica
\end{abstract}

\section{Introduction}

Femtosecond lasers are a single processing tool of transparent dielectrics allowing for the simultaneous integration of optical, fluidic, mechanical and sensing functionalities on the same chip [1]. Additionally, above the threshold for smooth material modifications and for suitable laser parameters, self-organized nanogratings are formed within the focal volume. The resulting form birefringence, with strength and direction controlled at each laser spot, allows for the fabrication of all-silica spatially variant phase plates, for optical vortexes generation or radially polarized beam converters. Up to now, nanogratings have only been observed in laser modified regions produced by fs-laser irradiation. Amplified fs-lasers have been the preferred choice for the above demonstrations.

Compared to the complexity of fs-laser systems, ps-lasers offer a much simpler and robust architecture. Despite the longer pulse duration thermal damage due to fast local heating melting and vaporization of the target is still avoided for pulse durations below 10-20 ps. Those facts have made ps-lasers an industry standard for precision micro-machining by surface ablation. However, it is still an open question whether ps-lasers are suitable for bulk machining of transparent dielectrics.

In this work we demonstrate the generation and control over strength and orientation of self-assembled nanogratings by 8 ps-pulses. Selective chemical etching in the laser exposed regions is also achieved thereby showing the possibility of bulk micro-machining of silica glass by ps-lasers. The performances of ps-machining is critically compared to fs-machining in terms of retardance induced by form-birefringence in the nanogratings, absorption and scattering losses, etching rate and homogeneity of the etched regions.

2. Sample preparation and experimental methods

A Nd: $\mathrm{YVO}_{4}$ laser delivering $8 \mathrm{ps}$ at the wavelength of $1064 \mathrm{~nm}$ was employed for laser inscription.
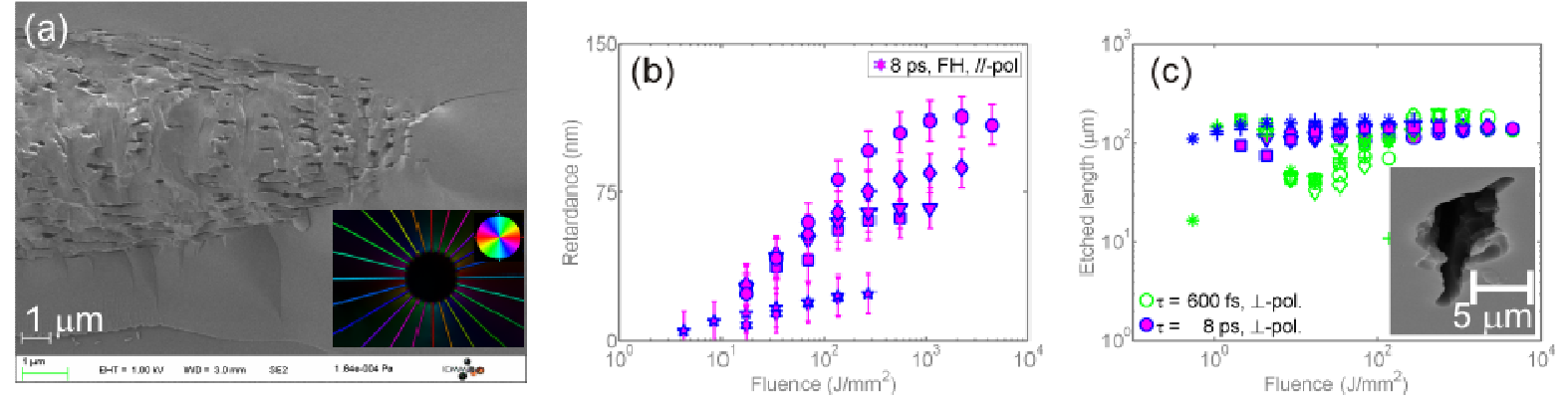

Fig. 1. (a) FEG-SEM, Secondary electrons image of a cleaved laser track in fused silica revealing the presence of nanogratings. Experimental conditions: $\lambda=1064 \mathrm{~nm}, \mathrm{NA}=0.65, \tau=8 \mathrm{ps}, \mathrm{E}_{\mathrm{p}}=1 \mu \mathrm{J}, f=200 \mathrm{kHz}, v=200 \mu \mathrm{m} / \mathrm{s}$. Laser was shining from the right end of the picture. Polarisation oriented perpendicular to the writing direction. (inset) Quantitative birefringence measurements of outwardly written laser tracks. Laser polarisation aligned parallel to the writing direction. $\lambda=$ $1064 \mathrm{~nm}, \mathrm{NA}=0.65, \tau=8 \mathrm{ps}, \mathrm{E}_{\mathrm{p}}=2 \mu \mathrm{J}, f=200 \mathrm{kHz}, v=200 \mu \mathrm{m} / \mathrm{s}$. Pseudo colours represent the angle of the slow axis of birefringence as indicated by the legend in the top right corner. (b) Measurements of the retardance vs. laser fluence in tracks processed by 8 ps-laser irradiation at $1064 \mathrm{~nm}$ for longitudinal polarisation. Symbols in (b) and (c) correspond to: (*) 12.5 kHz, (+) $25 \mathrm{kHz},(\square), 50 \mathrm{kHz},(\nabla) 100 \mathrm{kHz},(\diamond) 200 \mathrm{kHz},($ o) $400 \mathrm{kHz}$. Scanning speed $v=25-6400 \mu \mathrm{m} / \mathrm{s}$. (c) Etched length of the micro-channels formed in silica glass by ps- and fs-laser irradiation and 80 min in $2.5 \% \mathrm{HF}$ bath. Inset- representative SEM image of the aperture of a micro-channel made by ps-laser. 
The laser was focused $100 \mu \mathrm{m}$ below the surface of a $25 \times 25 \times 1 \mathrm{~mm}^{3}$ silica plate (SPECTROSIL) by means of a $20 \mathrm{X}$ aspheric lens of $\mathrm{NA}=0.5$. A series of lines was written by translating the sample transversely to the beam propagation direction. The pulse energy was $0.8 \mu \mathrm{J}$. For comparison a matching set of lines was written on the same sample by fs-laser (mode-locked regenerative amplified $\mathrm{Yb}: \mathrm{KGW}, \tau=600 \mathrm{fs}$ as measured with an autocorrelator). The experimental conditions chosen are well below the threshold for self-focusing.

Nanogratings were characterized by quantitative birefringence measurements (Cri Abrio imaging system). The system allows accurate measurements of the direction of the slow axis of birefringence and of the strength of the laser induced retardance. Subsequently to the laser exposure the sample was side-lapped and polished. Microchannels were formed by etching the sample in Hydrofluoric acid (HF) bath at $2.5 \%$ concentration for 80 minutes. The length of the etched channels was measured under bright illumination optical microscopy with a resolution of \pm $1 \mu \mathrm{m}$.

\section{Results and discussion}

The first evidence that form birefringence can be produced by pulses other than fs is inferred from quantitative birefringence measurements (Fig. 1(a)-inset). Control over the direction of the slow axis of the birefringence by the orientation of the laser polarization is shown. Further investigation by Field-Emission Gun Scanning Electron Microscopy (FEG-SEM) image of a cleaved laser track produced by ps-laser irradiation in fused silica provides direct and incontrovertible confirmation of nanogratings formation (Fig. 1.(a)). The FEG-SEM secondary electrons image shows the top of the cross-section of the laser damage with the nano-planes responsible for the form birefringence aligned perpendicular to the direction of the laser polarization (vertical in the figure). The periodicity is $\Lambda \sim 250 \mathrm{~nm}$ which is in line with nanogratings formed by fs-laser at similar pulse energies. A second periodicity, $\Lambda \sim \lambda / \mathrm{n} \sim 733 \mathrm{~nm}$, apparently increasing as we move away from the tip of the structure is observed along the horizontal direction. The strength of the retardance, defined as the product $R \equiv \Delta n \times L$ where $\Delta n$ is the overall birefringence and $L$ the optical path length, increases with the laser fluence (Fig. 1.(b)). The highest retardance, $(\mathrm{R}=$ $113 \pm 10 \mathrm{~nm}$ ), already enables writing quarter-wave plates for operation at $\lambda=355 \mathrm{~nm}$ and further improvement is possible by using lower NA lens, giving larger $L$ through the increased Rayleigh range. Despite the value of the induced retardance is comparable to what is obtained with fs-lasers the biggest hurdle to overcome for ps-laser fabricated wave-plates is posed by optical scattering and by the development of a stress regions surrounding the laser tracks resulting in rupture of the glass at high fluences as more and more lines are packed together to raster scan an area of interest.

Micro-channels formed by selective chemical etching of ps-irradiated laser tracks are demonstrated (Fig. 1.(c)inset). Data of the etched lengths after $80 \mathrm{~min}$ in diluted HF bath are plotted as a function of the laser fluence (Fig. 1.(c)). In the ps-regime etched lengths of about 120-150 $\mu \mathrm{m}$ are obtained in the whole range of fluences explored in this study $\left(0.5-4500 \mathrm{~J} / \mathrm{mm}^{2}\right)$. Incidentally this is irrespective of the direction of the polarization of the writing beam. The etching rate in both ps- and fs-laser tracks attains the same maximum value of $2 \pm 0.5 \mu \mathrm{m} / \mathrm{min}$ indicating that the limit to the process lies on the physical parameters of the substrate rather then on pulse properties. Generally, etching of ps-laser exposed regions leaves a coarse surface and an evident mismatch between the intensity profile of the laser beam and the contour of the micro-channel making it unsuitable for bulk machining.

In conclusion, we have provided the first evidence of self-organized nanogratings by ps-laser writing in silica. We have also shown selective chemical etching of ps-laser irradiated tracks. These results demonstrate the potential of ps-lasers for bulk machining of silica glass as an alternative to complex amplified fs-laser systems. The performance in terms of laser-induced retardance or etching rate is comparable to that obtained by fs-laser, however, challenges remain to be solved and may require different writing strategy. Despite pulses shorter than 10 ps and therefore below the limit for thermal damage in fused silica glass were employed raster scanning of adjacent lines almost inevitably results in deep cracks in the sample and in strong light scattering. Cracks could be likely avoided by heating the sample upto the glass softening temperature during laser irradiation. A potential advantage of using ps-lasers lies for those applications relying upon the generation of a stress field in the dielectric matrix such as for example the fabrication of optical waveguides in crystals.

\section{Acknowledgments}

This work is supported by the European Commission through the FP7 program: Femtoprint, NMP, project no 26010

\section{References}

[1] Y. Bellouard, A. Said, M. Dugan, and P. Bado, "Fabrication of high-aspect ratio, micro-fluidic channels and tunnels using femtosecond laser" pulses and chemical etching," Optics Express 12, 2120-2129 (2004)

[2] M. Beresna, M. Gecevicius and P.G. Kazansky "Polarization sensitive elements fabricated by femtosecond laser nanostructuring of glass", Optical Materials Express, Vol. 1 Issue 4, pp.783-795 (2011) 